\title{
Search for non-standard and rare decays of the Higgs boson with the ATLAS detector
}

\section{Roger Caminal Armadans*}

University of Illinois at Urbana-Champaign

E-mail: roger.caminal.armadansecern.ch

\begin{abstract}
Results of the latest searches for non-standard and rare decays of the Higgs boson with the ATLAS detector are presented. Good agreement is observed between data and the SM predictions in all searches, and the resuls are translated into upper limits on the branching fraction for the Higgs boson decaying into a $\phi$ meson and a photon, on the Higgs production cross-section times the branching fraction for the Higgs to decay into four $b$-quarks via two new light spin- 0 particles, and on the branching fraction for Higgs decaying into an electron or a muon and a tau lepton.
\end{abstract}

XXV International Workshop on Deep-Inelastic Scattering and Related Subjects

3-7 April 2017

University of Birmingham, $U K$

\footnotetext{
*Speaker.
} 


\section{Introduction}

Available measurements of Higgs boson properties from the ATLAS and CMS collaborations are so far consistent with a Standard Model (SM) Higgs with a mass of $125 \mathrm{GeV}$. Measurements of the production and decay rates of the Higgs boson and the constraints on its couplings yield an upper bound on the Higgs boson decay branching ratio to BSM particles of $0.34 \%$ at $95 \%$ confidence level (CL). This opens a new and rich experimental program that includes the search for exotic decays of the Higgs boson.

This document is not an extensive list of analyses, but a compilation of the latest ATLAS results on this topic. Section 2 describes the ATLAS search for a SM Higgs boson decaying to $\phi \gamma$. This decay is allowed in the Standard Model with a very small branching ratio and therefore an excess of events on this channel would be a clear indication for new physics. Section 3 describes the search for a Higgs boson decaying into two new spin- 0 particles, each of them further decaying into two $b$-quarks. The branching ratio for the decay $H \rightarrow 2 a \rightarrow 4 b$ is predicted to be relatively large in many models, thus motivating the search in this challenging final state. The search for lepton-flavor violating decays of the Higgs boson in final states involving electrons or muons, and a tau is described in Section 4. Finally, conclusions are provided in Sec. 5.

\section{Higgs boson decaying to $\phi \gamma$}

Rare decays of the $125 \mathrm{GeV}$ Higgs boson $H$ to a light meson and a photon $\gamma$ have been suggested as a probe of the Yukawa coupling of the Higgs boson to light $(u, d, s)$ quarks. While the Standard Model (SM) predicts these couplings to be small, substantial modifications are predicted in several scenarios beyond the SM (BSM). The decay of the Higgs boson to a $\phi$ meson and a photon would give access to the strange-quark Yukawa coupling and to potential deviations from the SM prediction $\left(\mathrm{BR}(H \rightarrow \phi \gamma)=(2.3 \pm 0.1) \times 10^{-6}\right)$. The corresponding decay of the $Z$ boson has also been considered, but will not be discussed in this document.

This Section describes the search for a Higgs boson decay to the exclusive final state $\phi \gamma$, where the $\phi$ meson is reconstructed from the decay $\phi \rightarrow K^{+} K^{-}$[1]. The search is performed with a sample of $p p$ collision data corresponding to an integrated luminosity of $2.7 \mathrm{fb}^{-1}$ recorded at a center-of-mass energy of $13 \mathrm{TeV}$ with the ATLAS detector [2]. In the absence of particle identification in the relevant momentum range, every reconstructed charged particle that satisfies quality requirements (e.g. on the number of hits in the silicon detectors) in events with at least 2 tracks of $p_{T}>400 \mathrm{MeV}$ originating from the primary vertex ${ }^{1}$ are assumed to be $K^{ \pm}$mesons. The $\phi$ candidates are reconstructed from pairs of opposite charged kaons with $p_{T}$ larger than $20 \mathrm{GeV}$ and $15 \mathrm{GeV}$, respectively, and with a mass consistent within $20 \mathrm{MeV}$ with the $\phi$ meson mass. Combinations of a $\phi \rightarrow K^{+} K^{-}$candidate and a photon are selected if they satisfy $\Delta \phi\left(K^{+} K^{-}, \gamma\right)>$ 0.5. The transverse momentum of the $\phi$ meson is required to be larger than a threshold that depends on the invariant mass of the system, to ensure optimal sensitivity.

The main source of background to the search comes from events involving inclusive multijet or photon+jet processes where a $\phi \rightarrow K^{+} K^{-}$candidate is reconstructed from tracks associated with a jet. The normalization of this background is extracted directly from a fit to data. The shape

\footnotetext{
${ }^{1}$ The primary vertex is defined as the vertx with the largest $\sum p_{T}^{2}$.
} 
is modeled with a nonparametric data-driven approach using templates to describe the kinematic distributions in a region with similar requirements to the signal region but with the isolation and some kinematic conditions relaxed.

The results are compared to background and signal predictions using an unbinned maximumlikelihood fit to the $m_{K^{+} K^{-}} \gamma$ distribution. Since no significant excess of data is observed with respect to the background, upper limits are set on the branching fractions for the Higgs boson decay to $\phi \gamma$ using the $\mathrm{CL}_{s}$ modified frequentist formalism with the profile-likelihood ratio test statistic. Figure 1 shows the result of the background-only fit. Confidence level (CL) upper limits on the branching fraction for $H \rightarrow \phi \gamma$ are computed. Values larger than $1.4 \times 10^{-3}$ are excluded at $95 \% \mathrm{CL}$, around 600 times the expected SM branching fraction.

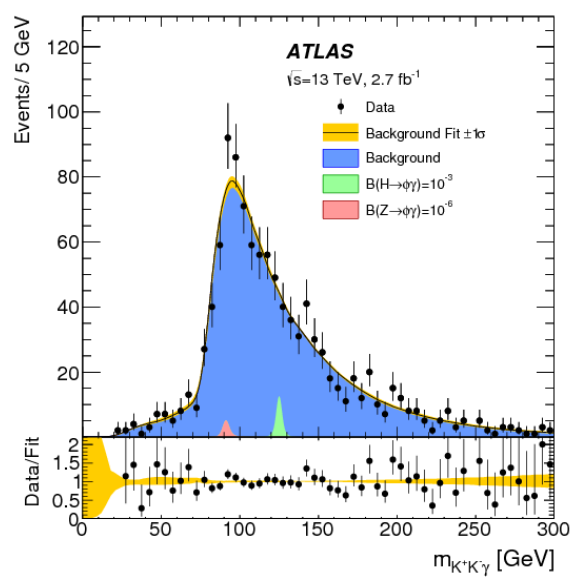

Figure 1: The $m_{K^{+} K^{-}} \gamma$ distributions of the selected $\phi \gamma$ candidates, along with the results of the maximum-likelihood fit with background-only model. The $1 \sigma$ uncertainty band corresponds to the total uncertainty of the background model. The Higgs and $Z$ boson contributions, expected for branching fraction values of $10^{3}$ and $10^{6}$, respectively, are also shown.

\section{New light resonances: Higgs boson decaying to two $a$-bosons}

Exotic decays are predicted by many theories of physics beyond the Standard Model, including those with an extended Higgs sector such as the Next-to-Minimal Supersymmetric Standard Model (NMSSM). One of the simplest possibilities is that the Higgs boson decays to a pair of new spinzero particles, $a$, which in turn decay to a pair of SM particles. The result presented in this section covers the unexplored decay mode in searches for $H \rightarrow a a$ by considering $a \rightarrow b b$ [3]. An example of a model with predominant $a \rightarrow b b$ decays is one where the new scalar mixes with the SM Higgs boson and inherits its Yukawa couplings. This search, performed on $p p$ collision data recorded with the ATLAS detector corresponding to an integrated luminosity of $3.2 \mathrm{fb}^{-1}$, focuses on the $p p \rightarrow W H$ process, with $W \rightarrow \ell v(\ell=e, \mu)$ and $H \rightarrow 2 a \rightarrow 4 b$ in the range $20 \mathrm{GeV}<m_{a}<60 \mathrm{GeV}$.

The $H \rightarrow 2 a \rightarrow 4 b$ decay chain is expected to have multiple $b$-jets, often three or four, satisfiying the object selection. The dominant background arises from $t \bar{t}$ events. Preselected events are required to have exactly one electron or one muon and at least three jets, of which two must be 
$b$-tagged. Events are then categorized into eight channels depending on the number of jets $(3,4$, and $\geq 5)$ and $b$-tagged jets (2, 3 and $\geq 4)$, referred to as $(n \mathrm{j}, m \mathrm{~b})$ indicating $n$ selected jets including $m b$-tagged jets. The categories most sensitive to the $H \rightarrow 2 a \rightarrow 4 b$ decay chain are $(3 \mathrm{j}, 3 \mathrm{~b}),(4 \mathrm{j}$, $3 \mathrm{~b})$ and $(4 \mathrm{j}, 4 \mathrm{~b})$, and are defined as signal regions. In order to improve the sensitivity of the search, several kinematic variables are identified to distinguish between signal and background, and are combined into multivariate discriminants based on boosted decision trees (BDT), trained in each of the signal regions. The distribution of the final discriminants in the eight analysis channels ${ }^{2}$ are combined into a global profile-likelihood fit to test the presence of a signal. Figure 2 (a) shows the best fit of the background predictions to data for the $(4 \mathrm{j}, 4 \mathrm{~b})$ signal region. No significant excess of data above the background prediction is found, and therefore upper limits are calculated for the quantity $\sigma(W H) \times \mathrm{BR}$, where $\mathrm{BR}=\mathrm{BR}(H \rightarrow a a) \times \mathrm{BR}(a \rightarrow b b)^{2}$ as function of $m_{a}$, as shown in Figure 2 (b).

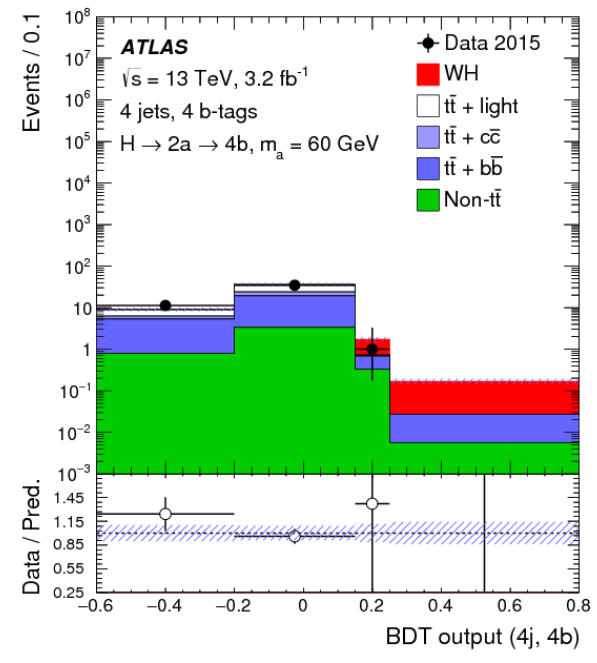

(a)

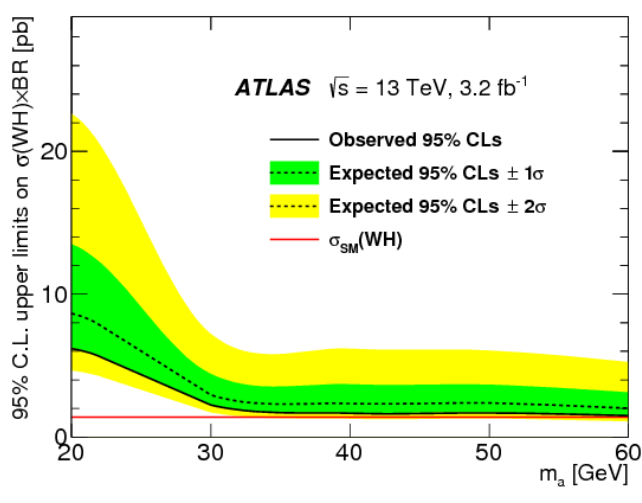

(b)

Figure 2: (a) Comparison between the data and prediction for the distribution of the BDT discriminant in the 4 jets $4 b$-tags signal region. This distribution after the fit is performed on data under the background-only hypothesis. The hashed area represents the total uncertainty in the background. The distributions for the signal model $W H, H \rightarrow 2 a \rightarrow 4 b$, with $m_{a}=60 \mathrm{GeV}$, are normalized to the SM $p p \rightarrow W H$ cross section, assuming $\mathrm{BR}(H \rightarrow a a) \times \mathrm{BR}(a \rightarrow b b)^{2}=1$. (b) Upper limit at $95 \%$ $\mathrm{CL}$ on $\sigma(W H) \times \mathrm{BR}$, where $\mathrm{BR}=\mathrm{BR}(H \rightarrow a a) \times \mathrm{BR}(a \rightarrow b b)^{2}$, versus $m_{a}$. The observed $\mathrm{CL}_{s}$ values (solid black line) are compared to the expected (median) $\mathrm{CL}_{s}$ values under the backgroundonly hypothesis (dotted black line). The surrounding shaded bands correspond to the $68 \%$ and $95 \%$ $\mathrm{CL}$ intervals around the expected $\mathrm{CL}_{s}$ values, denoted by $\pm 1 \sigma$ and $\pm 2 \sigma$, respectively. The solid red line indicates the SM $p p \rightarrow W H$ cross section, assuming $\mathrm{BR}(H \rightarrow a a) \times \mathrm{BR}(a \rightarrow b b)^{2}=1$.

\footnotetext{
${ }^{2}$ The BDT discriminants are used in each of the signal regions, while the scalar sum of the $p_{T}$ of the jets is used in the control regions.
} 


\section{Flavor violating couplings}

Lepton flavor violating (LFV) decays of the Higgs boson can occur in models with more than one Higgs doublet, composite Higgs models or models with flavour symmetries, among others. In this section two different complementary searches for LFV of the Higgs boson are presented [4]. The first study is a search for $H \rightarrow e \tau_{\text {had }}$ decays in the final state with one electron and one hadronically decaying $\tau$-lepton. The second analysis is a simultaneous search for the LFV $H \rightarrow e \tau_{\text {lep }}$ and $H \rightarrow \mu \tau_{\text {lep }}$ decays in the final state with a leptonically decaying $\tau$-lepton. These searches are based on the data sample of $p p$ collisions collected by the ATLAS detector at a center-of-mass energy of $\sqrt{s}=8 \mathrm{TeV}$ and corresponding luminosity of $20.3 \mathrm{fb}^{-1}$.

In the $H \rightarrow e \tau_{\text {had }}$ search, events with exactly one energetic electron and one opposite charge $\tau_{\text {had }}$ are classified in two signal regions with different electron and tau transverse mass requirements. Backgrounds for this signature can be classified in two major categories: events with true electron and $\tau_{\text {had }}$ candidates, and events with a misidentified $\tau_{\text {had }}$ signature. The first category is dominated by the irreducible $Z / \gamma^{*} \rightarrow \tau \tau$ production with some contributions from the $V V \rightarrow e \tau+X$ (where $V=W, Z$ ), $t \bar{t}$, single-top and SM $H \rightarrow \tau \tau$ production. The second category is dominated by $W+$ jets events with some contribution from multi-jet, diboson, $t \bar{t}$ and single-top. These backgrounds are estimated with data-driven techniques when possible, making use of several control regions enhanced in the different background processes [4].

On the other hand, the search for $H \rightarrow e \tau_{\text {lep }} / \mu \tau_{\text {lep }}$ selects events with exactly two oppositesign leptons, an electron and a muon. The lepton with the higher $p_{T}$ is indicated by $\ell_{1}$, and the other by $\ell_{2}$. The background estimation is based on the premise that the kinematic properties of the SM backgrounds are symmetric for electrons and muons, while the signal from LFV Higgs decays are not. For simplicity, the symmetry method is illustrated assuming a $H \rightarrow \mu \tau$ signal. In this scenario, the dilepton events in the dataset are divided into two mutually exclusive categories, a $\mu e$ sample ( $\ell_{1}$ is the muon, $\ell_{2}$ is the electron), and a $e \mu$ sample. With these assumptions, the SM background is expected to be split equally between the two samples ${ }^{3}$. The Higgs signal however, is only present in the $\mu e$ sample because the $p_{T}$ spectrum of electrons from the $H \rightarrow \mu \tau$ decays is softer than the muon $p_{T}$ spectrum. The final discriminant used in this search is the collinear mass, $m_{\text {coll }}$, defined in Ref. [4].

The results of the individual searches for LFV $H \rightarrow e \tau$ and $H \rightarrow \mu \tau$ decays in the $\tau_{\text {had }}$ (including the result from Ref. [5] for $H \rightarrow \mu \tau$ ) and $\tau_{\text {lep }}$ channels are statistically combined. No data excess has been found with respect to the SM background prediction. Upper limits on the Branching ratio for $\mathrm{BR}(H \rightarrow e \tau)$ and $\mathrm{BR}(H \rightarrow \mu \tau)$ are shown in Figure 3 .

\section{Conclusions}

This contribution presents the results of searches for non-standard and rare decays of the Higgs boson with the ATLAS detector, obtained from proton-proton collision data at center of mass energies of $8 \mathrm{TeV}$ and $13 \mathrm{TeV}$. Good agreement is observed between data and the SM predictions in all cases, and the results are translated into upper limits on different scenarios for new physics.

\footnotetext{
${ }^{3}$ Effects on asymmetries produced by misidentified non-prompt leptons affecting differently electrons and muons, as well as effects from the trigger efficiency and reconstruction are taken into account
} 


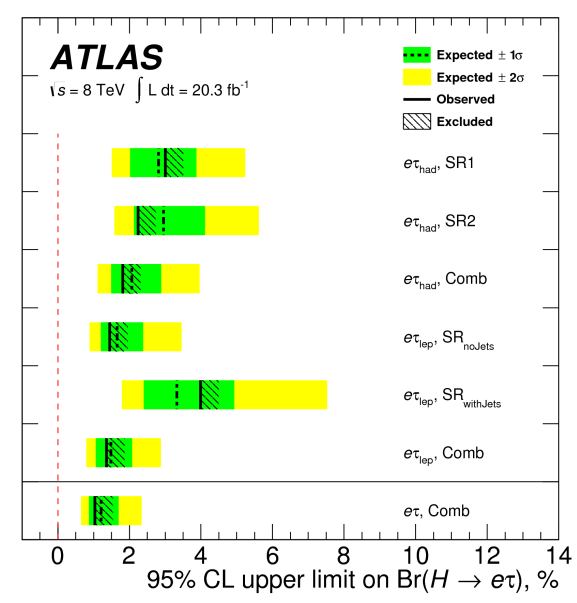

(a) $H \rightarrow e \tau$

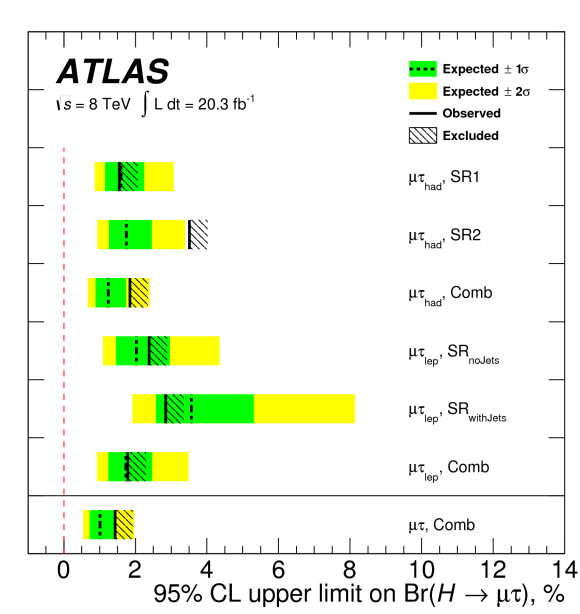

(b) $H \rightarrow \mu \tau$

Figure 3: Upper limits on LFV decays of the Higgs boson in the $H \rightarrow e \tau$ hypothesis (a) and $H \rightarrow \mu \tau$ hypothesis (b). The limits are computed under the assumption that either $\operatorname{BR}(H \rightarrow \mu \tau)=0$ or $\operatorname{BR}(H \rightarrow e \tau)=0$. The $\mu_{\text {had }}$ channel is from Ref. [5].

\section{References}

[1] M. Aaboud et al. [ATLAS Collaboration], "Search for Higgs and $Z$ Boson Decays to $\phi \gamma$ with the ATLAS Detector," Phys. Rev. Lett. 117 (2016) no.11, 111802 doi:10.1103/PhysRevLett.117.111802 [arXiv: 1607.03400 [hep-ex]].

[2] G. Aad et al. [ATLAS Collaboration], "The ATLAS Experiment at the CERN Large Hadron Collider," JINST 3, S08003 (2008).

[3] M. Aaboud et al. [ATLAS Collaboration], "Search for the Higgs boson produced in association with a $W$ boson and decaying to four $b$-quarks via two spin-zero particles in $p p$ collisions at $13 \mathrm{TeV}$ with the ATLAS detector,” Eur. Phys. J. C 76 (2016) no.11, 605 doi:10.1140/epjc/s10052-016-4418-9 [arXiv:1606.08391 [hep-ex]].

[4] G. Aad et al. [ATLAS Collaboration], "Search for lepton-flavour-violating decays of the Higgs and $Z$ bosons with the ATLAS detector," Eur. Phys. J. C 77 (2017) no.2, 70 doi:10.1140/epjc/s10052-017-4624-0 [arXiv:1604.07730 [hep-ex]].

[5] G. Aad et al. [ATLAS Collaboration], JHEP 1511, 211 (2015) doi:10.1007/JHEP11(2015)211 [arXiv:1508.03372 [hep-ex]]. 